\title{
From a classical neuroscience towards a quantum theory of mind and consciousness $^{\text {th }}$
}

\author{
Alexander V. H. McPhail ${ }^{\mathrm{a}}$ \\ 29 March 2009 \\ Natural Philosophy: 003 \\ (c) Lucid Press 2009. All Rights Reserved. \\ a $1 \mathrm{H} / 18$ Federal Street, \\ Auckland Central, \\ Auckland 1010, \\ AOTEAROA
}

\begin{abstract}
An overview of the western progress towards a theory of mind and consciousness. An evolutionary argument for the physical reality of subjective experience is supported by recent work in high temperature quasiparticle Böse condensates.
\end{abstract}

Homo sapiens (humans) seek self-knowledge. Correct understanding of what humans are can lead to increased longevity, improved quality of life, and personal satisfaction. By studying the biology of man, diseases can be cured or ameliorated, thus reducing their contribution to the overall mortality rate. Having information about beliefs and desires and how they operate can guide ethical theories and the behaviour of groups and individuals. Insight into what a subject that feels and can contemplate itself is would fill a philosophical void. The mind has been described as what the brain does. Consciousness is a key aspect of the mind.

\section{The Brain}

Humans are an animal species of the kingdom Animalia. Animals are colonies of eukaryotic cells that live, perceive, and exhibit voluntary action. These colonies evolved from single-celled eukaryotes that began to cohere and communicate, eventually leading to specialisation and the development of organs and complex body forms. Eukaryotes are thought to have been nucleated cells that acquired endosymbionts such as mitochondria. Prokaryotes are simple cells that lack a nuclear envelope and predate eukaryotes (Arms and Camp, 1995). According to the RNA world hypothesis, self-replicating molecules acquired fatty membranes and the ability to manufacture both these membranes and other necessary metabolites (Orgel, 1994). The driving force behind this evolution from simple molecules is the generation and replication of mutations in populations whose sizes are limited by the scarcity of resources, thus favouring the selection of species that survive and reproduce efficiently and rapidly (Russell, 1998). A constant flow of energy from sources such as the sun fuel these chemical reactions, which create complex, ordered structures from relative chaos (Eigen and Schuster, 1979). These chemical reactions obey the second law of thermodynamics and are, so it is believed, completely governed by the rules of QED, which determine the shape and charge of molecules (Voet and Voet, 2004, Feynman, 1985).

\footnotetext{
This paper was the beginning of the introduction for a never-to-be-completed 2004 Masters in Biosciences thesis at the Victoria University of Wellington entitled Consciousness, Pain, and Opiates. Opioid receptors, according to my flourescence in situ hybridisation results appear throughout the Rattus norvegicus encephalus, which cannot eliminate the hypothesis that opioid receptors act upon condensate quasi-particles to mode-shift the experienced quale, expressible both as a quantum operator and on an experiential axis. Differential colocalisation of different subtypes and different metabotrobic species might indicate differing modal strengths in different regions for various axes of qualia.

Email address: vivian.mcphail@gmail.com (Alexander V. H. McPhail)
} 
Animal behaviour is directed by an organ, the brain. Some communication in the animal is achieved by the transmission throughout the organism of hormone molecules from various cell tissue types, such as the pancreas and pituitary gland of the brain. However, there is a need for organisation on a faster scale, and thus the ability to translate stimulus into action on an almost instantaneous basis has evolved. Sensory organs like the skin, tongue, eyes, ears, and intestinal tract transmit signals to the brain. Muscles have sensory nerves which indicate the state of the muscle and thus the positions of the limbs. These afferent pathways are mirrored by efferent pathways from the brain which activate motor control of muscles. There are some reflex arcs which extend only as far as the spinal chord, such as the response to a doctor's hammer on the knee. Apart from these basic reflexes, all the nerve signals travel through the brain (Ganong, 1995).

The brain is composed of nerve cells and glial cells. Glial cells essentially perform a support role for the nerve cells. Brain cells are organised in a modular fashion, with different structures in the brain fulfilling different functions. The brain is divided into six major sections, medulla, pons, cerebellum, midbrain, diencephalon, and telencephalon (cerebral hemispheres). The medulla connects directly to the spinal chord, and is involved in controlling respiration and heart rate, as well as having some relay nuclei. The pons has structures involved in respiration, taste, and sleep and also relays information from the cerebral cortex to the cerebellum. The midbrain is important for motor control. The cerebellum is involved in fine motor control, learning motor skills, coordination and posture, and some cognitive functions. The diencephalon comprises the thalamus and hypothalamus. The thalamus is an important relay and gating station for sensory and motor information sent to the cerebral hemispheres. The thalamus is also thought to have regions involved in arousal and consciousness. The hypothalamus is concerned with homeostasis and reproduction by controlling the pituitary gland and being connected to much of the nervous system. Also, the hypothalamus initiates and maintain rewarding behaviours. The cerebral hemispheres comprise the cortex, basal ganglia, amygdala, and hippocampal formation and are involved in perceptual, motor, and cognitive functions as well as emotion (amygdala) and memory (hippocampus) (Kandel et al., 2000).

Nerve cells transmit signals. Incoming signals are received at the dendrite and summated across the soma into an action potential which travels down the axon of the neuron to the synaptic buttons, which stimulate downstream neurons or tissue. The synapses release a chemical neurotransmitter which traverses the synaptic cleft and interacts with receptor molecules on the post-synaptic membrane. These ionotropic receptor molecules respond by opening pores which are selectively permeable to specific ion species, $\mathrm{NA}^{+}, \mathrm{K}^{+}$, and $\mathrm{CL}^{-}$. The ions rush through the membrane, depolarising it, thus propagating an electrical signal a short distance across the cell surface. A depolarisation can affect nearby voltage-sensitive gates, thus depolarising an adjacent region. This travelling wave may cross the soma membrane and reach the axon hillock. If there is sufficient strength to depolarise the hillock an all-or-none signal is propagated in the same fashion down the axon. When the action potential reaches the synapses the membrane depolarisation opens calcium channels which let $\mathrm{CA}^{2+}$ ions rush into the synapse and facilitate exocytosis of vesicles containing receptor molecules. This propagates a signal from one nerve to the next (Kandel et al., 2000).

Neuronal functioning can be modulated. On the cellular surface of neurons there are also receptor molecules which perform modulatory functions. These metabotropic receptors either affect the membrane polarisation on a longer time scale, by activating ion channels, or initiate secondary messanger cascades within the cell, which can change cellular function and gene expression. The molecules which affect these receptors can be coreleased with the primary neurotransmitter or are hormones diffused from surrounding tissue or the blood-stream (Kandel et al., 2000).

Networks of neurons can change, and thus adapt to the environment. The number of synapses connecting one axon to downstream dendrites is subject to change. The changes are brought about by the addition or removal of synapses through remodelling of the cellular architecture. Also, the number of post-synaptic receptor molecules can increase or decrease. Together, these factors afford the neuronal structure and function of the brain some plasticity. This plasticity can be used to change the response of an organism to its environment and is manifest as the phenomenon of learning (Kandel et al., 2000).

The brain has about $10^{11}$ neurons. If each of $N$ neurons were connected to each other there would be $N^{2}$ connections, thus there would be about $10^{22}$ synapses. However, there are only approximately $10^{14}$ synapses in the brain, reflecting a modular, non-global structure. Study of artificial neural networks using a mathematical model of the neuron as a summation and threshold device shows that any computable function can be encoded in a three layer network, which comprises an input layer, a hidden layer, and an output layer, with sufficient nodes in each layer. There is a back-propagation of error algorithm which allows these network topologies to learn any pattern of inputs and outputs (Russell and Norvig, 1995). This suggests that the brain has the raw information-processing power to eventually learn 
or evolve the correct response to any particular environmental stimulus.

\section{The Mind}

The ancient Egyptians are thought to be the first civilisation to immortalise the soul (Herodotus, 440BCE). Plato was the first Greek philosopher to do likewise (Plato, $347 \mathrm{BC}$ ). Aristotle remained agnostic on the question, but did leave the door open (Aristotle, 350BCE). Christian thinkers developed a complex set of arguments on the existence of a single god. Descartes had a conviction of faith in the existence of God and the immortality of the soul, which he sought to justify philosophically. He divided existence into two realms, the physical and the mental. His famous dictum cogitō ergo sum, I think therefore I am, sought to illustrate the existence of a mental realm purely from the existence of thought. Even if experience were a dream, it would still exist (Descartes, 1641). Descartes identified the self as single, simple, and continuing. However Hume argued that upon introspection there was no such identifiable notion. A paradoxical argument that Descartes was correct in asserting such a self and Hume was right in saying that it cannot be experienced as anything but a vacuous symbol was put forward by Kant. Shear (1999) likens this state of pure consciousness to one that is reached through various meditative techniques. A dualistic philosophy of mind is one which maintains this Cartesian separation of mind and body.

The mind is a term used by humans to describe aspects of self such as personality, thought, reason, memory, intelligence, emotion, and self-awareness. Whether there is a particular entity over and above the collection of particular thoughts and feelings is an open question. The modern, rational scientist attempts to describe and explain phenomena within the confines of the physical conception of the universe. Such materialist theories come in a number of varieties. An identity theory holds that pain, for example, is merely the firing of a nociceptive fibre, that there is a direct correspondence between mental properties and physical properties. Functionalists argue that what are called mental events are merely functional, they are a causal link between inputs, other mental states, and outputs and have no autonomous existence beyond the brain (McPhail, 2007). The notion that psychological terms and concepts can be completely recast in the terms of more fundamental neuroscience is a reductionist notion. These eliminative materialist theories typically try to maintain a completely objective account of the mind and discredit the simple observation that humans experience mental events subjectively.

Many of the properties of the mind can be viewed as being representational. This includes signs for the state of the environment, such as the various pathways involved in visual and aural processing, and the state of the individual, including the body-map, memory, and mood. The information-processing ability of the brain is possibly sufficient to explain the behaviour of animals with respect to the linking of inputs and outputs. However, introspective, communicating humans recognise an additional faculty when inspecting themselves. This is the dimension of feeling. Information-processing, or the manipulation of symbols, does not necessarily generate anything that can be thought of as a feeling. It must be remembered that information is the contextually decoded interpretation of data. Thus the information is semantically internal to a subjective observer. Current neuroscience explains the information-processing element of mind, but cannot describe or explain how feelings and impulses are generated. Thus, an eliminative position has discarded the subjectivity of experience in trying to explain the mind.

\section{Consciousness}

One aspect of mind is consciousness. While the property of self-awareness, the experience of a self-representation, is no doubt an important feature along the evolutionary scale, it is distinct from the primacy of experience. There are certain mental events which are synonymous with direct experience. The smell of a rose, the taste of fruit, the pain of a stubbed toe are all examples of what philosophers call qualia. These experiences are felt and experienced subjectively. There is something that it is like to be a human (Nagel, 1974). This apparent unity of consciousness presents a problem, known as the binding problem. There must be a mechanism in the brain by which all the disparate bits of information and feelings are combined into the perceived unity. The two features of consciousness anomalous with respect to the current understanding of physics are the presence of qualia and the subjective unity of experience.

Dennett attempts to explain away qualia has having no significance. He describes his target as private, ineffable, intrinsic, and subjective. Through a series of intuitive arguments, Dennett attempts to convince that what might seem obvious is nonetheless misguided and confused (Dennett, 1988). The Cartesian Theatre is the term used by Dennett 
to describe the view that there is a homunculus that directs the mind. This view forces an infinite regress, as each homunculus requires their own homunculus to explain their behaviour. Instead, Dennett hypothesises that there are societies of simple homunculi, for example edge detectors, which are run by some virtual machinery. This computer directs the various homunculi into a stream of consciousness which has no seat of perception. Consciousness is described as an emergent property of computation (Dennett, 1991). Thus Dennett has explained away the physical aspect of qualia and the apparent unity of consciousness. This view fails to explain the directly observable reality of experience, and the apparent unity of that experience. One attempt to explain away the homuncular theory sets up an image of a regress of identical but metrically reduced homunculi, perhaps this is a failure of imagination as an internal decision-making executive would not need miniature eyes and miniature thumbs to drink a miniature container of watery stuff.

Churchland (2002) criticises the functionalist perspective because it relies upon the emergence of consciousness from a software system irrespective of the hardware upon which it runs. Instead, consciousness is described as being tied to the level of meta-representations developed in the brain. There is a distinction between self and non-self, and the inner representations of the self and the external environment. According to this theory, consciousness is somehow an emergent property of complicated self-reference. Unfortunately, this approach has ignored the quality of subjective experience, and also fails to explain the generation of qualia by information.

Jackson (1982) believes that the physicalist description of the universe leaves qualia out of the picture. Once we have all the physical information about the structure of the visual system, we still do not know what it is like to experience red. Thus he argues that qualia exist. However, he maintains that qualia are epiphenomenal, they are causally inefficacious. Qualia are supposed to be a mere by-product of the evolution of biologically advantageous structures. As by-products, mental states have no ability to effect change in the world, seemingly resigning observers to curious onlookers. There is no reason that evolutionary by-products are necessarily acausal and the argument that qualia are not evolutionarily advantageous is weak. The problem with causal qualia is that a mechanism by which this energy or force is transferred has not been elucidated and seems to escape physical determinism (but see below (McPhail, in prep)).

Searle (1998) argues that consciousness is a physical product of the functioning nervous system, and is thus amenable to scientific research. He distinguishes the ontological subjectivity of consciousness from the epistemic objectivity of science and argues that scientific objectivity can be maintained in the study of a subjective phenomenon. He also points out the problem with pure information-processing systems and defends the qualitative subjectivity of experience, the existence of qualia.

Qualia are physical phenomena with causative power. Pain feels negative and sex feels positive. These feelings correlate with biological advantage, with pain to be avoided and sex to be enjoyed. The ripeness of fruit and the associated benefit to the individual is also associated with positive or negative experiences mediated by the taste organs. Thus, there is a correspondence between biological advantage and the axes of qualia. There would seem to be a strong evolutionary advantage to having an instantaneous indication of the cost or benefit of a particular behaviour. Qualia provide such an indication. In order for evolution to operate on qualia there must be a mechanism by which the genetic material can affect qualia. This, presumably, is through the determination of the structure and function of nervous tissue by DNA, which affects the components of the brain which generate the qualia. More interestingly, for the presence of qualia, and thus consciousness, to be selectively positive, they must have some effect upon the behaviour of the individual, so that there may be some selective pressure between behaviours. If qualia were epiphenomenal then they would be evolutionary coincidences, but then they would not necessarily mirror the evolutionary cost or benefit of stimuli as well as they do. For example, there is an extremely close fit between the reflective properties of surfaces and the colour quale that is perceived, colour associations are constant. Thus qualia are affected by the physical world, and conversely, have an effect upon the physical world.

Consciousness aids learning, and thus provides evolutionary advantage. The unity of consciousness may act to compare different modalities instantaneously. Awareness of coincidences between modalities can lead to improved learning. There might be an evolutionary or ontogenetic crawl, in that some problems which used to be solved by conscious processes are superceded by automatic processes.

Consciousness is not associated with the entire brain. The contents of primary visual cortex are not available to immediate consciousness (Crick and Koch, 1995). Inhibition of stimulatory filtering by psychotropics such as $d$ lysergic acid can reduce the coherence of experienced inputs yet do not remove the overlaying of feature modules such as colour sensation and edge detection. Blindsight is a condition in which the subject reports having no visual stimulus 
in a specific field yet can still use information from that field in forced-choice guesses. There are two major high level visual streams, the dorsal and the ventral. It appears that the dorsal stream is non-conscious and involved in more automatic behaviours, whereas the ventral stream is conscious (Hameroff et al., 1996). Furthermore, from studies of brain injuries it is more clear that certain regions of the brain do not directly affect consciousness. For example, stroke damage to the hippocampus can destroy explicit memory, however the subject can still recall distant memories and still maintains consciousness. Damage to regions of the cerebral hemispheres can affect highly specific abilities, such as the ability to understand grammatical constructions (Wernicke's aphasia), and the ability to generate speech (Broca's aphasia), without affecting consciousness (Kandel et al., 2000). On the other hand, the Ras extends upwards to the thalamus and is involved in controlling levels of arousal. The thalamus has many re-entrant pathways to and from the particular regions of the cortex and is the most likely candidate for the location of the neuronal correlate of consciousness. Cortical cells have also been suggested as being involved in consciousness.

Crick and Koch (1990) point out that EEG oscillations between 25 and $95 \mathrm{~Hz}$, called gamma oscillations, are candidates for the neural correlates of consciousness. This synchronisation has been put forward as a solution to the binding problem, cells that fire together refer to the same entity. These oscillations are associated with re-entrant thalamocortical networks, but are not generated by axonal spiking. Dendro-dendritic cells in the cortex with electrical gap-junctions appear to be the generators of the $40 \mathrm{~Hz}$ oscillations. This synchrony might be just to stimulate the subsequent thalamic cells synchronously, whose firing is more closely linked with consciousness. EEG recordings of experienced meditators contemplating pure consciousness have increased gamma oscillations and a strengthened non-meditative gamma baseline. Pockett (2000) hypothesises that consciousness is epiphenomenal and associated with a certain type of spatiotemporal pattern of the brain's EM field. The organised EM field is dependent upon the synchronised firing of neurons, as individual neuronal firing is not sufficient for the generation of wide-scale EM fields. McFadden (2002b) adds to such a theory and postulates that the state of Em field can affect firing of neurons by downloading information (McFadden, 2002a). It should be noted that the magnetic component of an EM field is capable of storing energy. An Em field might generate individual qualia, but still does not explain the subjective unity of consciousness. An EM field is reducible to the transmission of virtual photons, thus there is no identifiable unity (Feynman, 1985). The attempt to explain the unity of consciousness has led to the speculation that consciousness could be related to a bizarre state of matter, a BEC (Fröhlich, 1968, Cairns-Smith, 1996, Giamarchi et al., 2008). In this phase, more than one particle share the same quantum state, thus information becomes global. The frequency of the oscillations might be related to requirements for the achievement of a quantum coherence between certain elements of the pulsing neurons.

NMDA receptors are implicated in the mechanism of neural plasticity. They are coincidence detectors which cause an influx of $\mathrm{CA}^{2+}$ ions when two conditions are met, that the membrane has depolarised, which removes $\mathrm{MG}^{2+}$ ions from the channel pore, and the receptor has been bound by a glutamate molecule, which allows the ions through. LTP and LTD, an increase and decrease of synaptic number, are dependent upon the $\mathrm{CA}^{2+}$ influx caused by NMDA receptor activation (Nishiyama et al., 2000, Nakazawa, 2002). NMDA receptors have been implicated in the study of consciousness as they are affected by many anaesthetics and although there are brain regions with these receptors and not consciousness, nevertheless, NMDA receptors are prevalent in brain regions most closely associated with consciousness. Rocha (da Rocha et al., 2001) suggests that the $\mathrm{CA}^{2+}$ molecules trigger intracellular cascades which provide energy for quantum computation in either $\mathrm{H}$ or $\mathrm{C}$ atoms of particular intracellular molecules. Their theory is highly dependent upon the synchronisation of neural assemblages, especially in the corticothalamic re-entrant pathways, and relies upon specific molecules in disparate regions of the brain to be entangled.

There are some theories of consciousness which rely on quantum computation. Computation in classical computers is composed of the manipulation of binary states 0 and 1 . In a quantum computer these states can be superposed so that the actual state is a probabilistic mixture of both states. This feature allows simultaneous computations of different options, which would have to be computed serially in a classical machine. This parallelisation of computation can lead to rapid processing of multiple inputs (Shor, 1997), and this could explain the integration of multiple inputs simultaneously by consciousness. However, this computational ability still falls short of explaining the subjective experience of consciousness for the same reason as the classical computer, information processing does not necessarily give rise to subjectivity. Also, quantum computation is still computation which eventually resolves into a classical token.

Penrose (1989) believes that there is a certain non-computable aspect to thought, as typified by the inductive reasoning of a mathematical genius. He uses this observation to suggest that there is a quantum aspect to the mind as 
well as the neuroscientific information processor. Penrose suggests an interpretation of quantum mechanics in which gravity interacts with the state of a superposed quantum particle. Essentially gravity acts to spontaneously collapse the wave function when a certain balance of spatial extension has been passed. This theory remains silent on the orchestrating force which is supposed to coincide with free will. If free will exists then there must be some motive power that eventually triggers neuronal impulses. We have hypothesised that qualia are causal, and if so there must be some such mechanism. If the only motive force is that generated by the collapse of the wave function triggered by gravity, and this also generates qualia, then what is perceived as free will is merely the product of quantum noise and the physical structure of the nervous system.

Anaesthetics, which selectively ablate consciousness, are gaseous compounds that are thought to interact by van der Waal forces with hydrophobic pockets in certain cellular proteins, affecting their structure and thus their function. The most sensitive neural proteins are GABAergic, glycinergic, serotinergic, and nicotinic acetylcholine receptors. The function of some receptors is dependent upon the integrity of cellular microtubules. One theory is that anaesthetics interfere with the ability to generate a macroscopic quantum coherence or the ability for the quantum superpositions of electron dipoles to effect computation (Hameroff, 1998, Hameroff and Penrose, 1996a,b).

Chalmers (1995) argues that quantum computation per se falls to the same arguments as classical computers, it does not explain what he calls the hard problem of consciousness. This is the problem of explaining how subjective experience arises, and is different in kind from other cognitive mechanisms explicable in terms of information processing. He proposes that consciousness is a fundamental property of the universe, just like mass, charge, spin, and space. As a fundamental, Chalmers introduces the dual aspect theory of information, that at least some information has both a physical aspect and a phenomenal aspect. This does not explain how the phenomenal aspect arises, but seems to allow the physical aspect to generate the phenomenal. Chalmers view is reconcilable with a materialist view of the universe, as long as the view is open to a paradigm shift in physical ontology. According to such a theory, the universe contains proto-conscious entities which interact to yield consciousness. Schrödinger (1945) mentions the Upanishads as having such a panpsychic view. McPhail (in prep) lays the groundwork for a theory of mind based on organisations of energetic quasi-particles arising from collective modes of energy storage driven by not quite constitutive $\mathrm{Na}^{+} / \mathrm{K}^{+}$ pumps.

\section{Acknowledgements}

My mother, Alicia de Joux, for demonstrating courage. Douglas Hofstadter for Gödel, Escher, Bach. Dr. Darren Day for allowing an interest beyond classical biochemistry. Prof. Kim Sterelny for initial signposts into the philosophy of mind.

\section{References}

Aristotle (350BCE). On the Soul.

Arms, K. and Camp, P. S. (1995). Biology, 4th edn, Saunders College Pub., Fort Worth

Cairns-Smith, A. G. (1996). Evolving the Mind, Cambridge University Press, Cambridge.

Chalmers, D. J. (1995). Facing up to the problem of consciousness, Journal of Consciousness Studies 2(3): 200-219.

Churchland, P. S. (2002). Self-representation in nervous systems, Science 296: 308-310.

Crick, F. and Koch, C. (1990). Towards a neurobiological theory of consciousness, Seminars in the Neurosciences 2: 273-304.

Crick, F. and Koch, C. (1995). Are we aware of neural activity in primary visual cortex, Nature 375: 121-123.

da Rocha, A. F., Jr., A. P. and Coutinho, F. A. B. (2001). N-methyl-D-aspartate channel and consciousness: from signal coincidence detection to quantum computing, Progress In Neurobiology 64: 555-573.

Dennett, D. C. (1988). Quining qualia, in A. Marcel and E. Bisiach (eds), Consciousness in Modern Science, Oxford University Press, Oxford.

Dennett, D. C. (1991). Consciousness Explained, Little, Brown and Co., Boston.

Descartes, R. (1641). Meditations.

Eigen, M. and Schuster, P. (1979). The Hypercycle, Springer-Verlag, Berlin.

Feynman, R. P. (1985). QED: the strange theory of light and matter, Princeton University Press, Princeton.

Fröhlich, H. (1968). Long-range coherence and energy storage in biological systems, International Journal of Quantum Chemistry 2: 641-649.

Ganong, W. F. (1995). Review of Medical Physiology, 17th edn, Appleton \& Lange, Norwalk.

Giamarchi, T., Rüegg, C. and Tchernyshyov, O. (2008). Böse-Einstein condensation in magnetic insulators, Nature Physics 4(9): 198-204.

Hameroff, S. and Penrose, R. (1996a). Orchestrated reduction of quantum coherence in brain microtubules: A model for consciousness?, in Hameroff et al. (1996), pp. 507-540.

Hameroff, S. R. (1998). Anesthesia, consciousness and hydrophobic pockets - a unitary quantum hypothesis of anesthetic action, Toxicology Letters 100-101: 31-39. 
Hameroff, S. R., Kasniak, A. W. and Scott, A. (eds) (1996). Toward a Science of Consciousness I, The MIT Press, Cambridge.

Hameroff, S. R. and Penrose, R. (1996b). Conscious events as orchestrated spacetime selection, Journal of Consciousness Studies 3(1): 36-53.

Herodotus (440BCE). The History of Herodotus.

Jackson, F. (1982). Epiphenomenal qualia, Philosophical Quarterly 32: 127-36.

Kandel, E. R., Schwartz, J. H. and Jessell, T. M. (eds) (2000). Principles of Neural Science, 4th edn, McGraw-Hill, New York.

McFadden, J. (2002a). The conscious electromagnetic information (cemi) field theory, Journal of Consciousness Studies 9(8): 45-60.

McFadden, J. (2002b). Synchronous firing and its influence on the brain's electromagnetic field, Journal of Consciousness Studies 9(4): 23-50.

McPhail, A. V. H. (2007). On mad pain and martian pain: My experienced pain is concrete — even more trouble with functionalism, web log.

URL: $\quad$ http://soloqi.wordpress.com/2007/09/18/on-mad-pain-and-martian-pain-my-experienced-pain-is-concrete-even-more-trouble-withfunctionalism/

McPhail, A. V. H. (in prep). Quantum teleportation and mental telepathy, Natural Philosophy .

Nagel, T. (1974). What is it like to be a bat?, The Philosophical Review 83: 435-450.

Nakazawa, K. (2002). Requirement for hippocampal CA3 NMDA receptors in associative memory recall, Science 297: 211-218.

Nishiyama, M., Hong, K., Mikoshiba, K., ming Poo, M. and Kato, K. (2000). Calcium stores regulate the polarity and input specificity of synaptic modification, Nature 408: 586-588.

Orgel, L. E. (1994). The origin of life on the earth, Scientific American pp. 77-83.

Penrose, R. (1989). The Emporer's New Mind, Oxford University Press, Oxford.

Plato (347 BC). The Republic, Heron Books, London.

Pockett, S. (2000). The Nature of Consciousness: A Hypothesis, Writers Club Press.

Russell, P. J. (1998). Genetics, 5th edn, Benjamin/Cummings, Menlo Park.

Russell, S. J. and Norvig, P. (1995). Artificial Intelligence: A Modern Approach, Prentice Hall, Upper Saddle River.

Schrödinger, E. (1945). What is Life?: the physical aspect of the living cell, Cambridge University Press, Cambridge.

Searle, J. R. (1998). How to study consciousness scientifically, Philosophical Transactions of the Royal Society, London. B 353: 1935-1942.

Shear, J. (1999). Experential clarification of the problem of self, The MIT Press, Cambridge, pp. 475-488.

Shor, P. W. (1997). Polynomial-time algorithms for prime factorization and discrete logarithms on a quantum computer, SIAM J.Sci.Statist.Comput 26: 1484 .

Voet, D. and Voet, J. G. (2004). Biochemistry, 3rd edn, J. Wiley and Sons, Hoboken. 